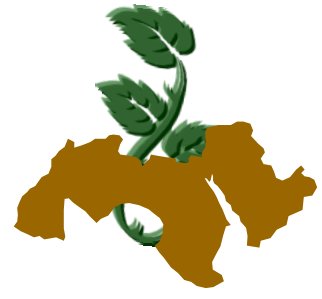

Arab Univ.

J. Agric. Sci., Ain Shams Univ., Cairo, 18(1), 181-189, 2010

\title{
NUTRITIONAL STATUS OF FOSTER GRAPEFRUIT IN SUDAN CENTRAL CLAY PLAIN
}

\author{
Elhassan 1, A.A.M.; A.M.A. El-Tilib²; A.A. Hashim'; H.S. Ibrahim¹ \\ and A.H. Awadelkarim ${ }^{2}$ \\ 1- Agricultural Research Corporation (ARC), Wad Medani, Sudan \\ 2- Department of Soil and Environment Sciences- Faculty of Agriculture-Shambat-PC 13314, \\ Khartoum, Sudan
}

Keywords: Foster grapefruit, Sennar area, Soil conditions, Management practices

\begin{abstract}
A detailed survey was carried out for 8 Foster grapefruit (Citrus paradisi Macf.) groves in Sennar area $\left(13^{\circ} 37^{\prime} \mathrm{N}, 33^{\circ} 37^{\prime} \mathrm{E}\right)$ representing Sudan Central Clay Plain. Trees were budded on Sour orange (Citrus aurantium L.). Soil and leaf samples were collected and analyzed according to standard procedures. Soil analyses included particle size distribution, $\mathrm{pH}$ (paste), ECe, SAR, N, P, K, Ca, $\mathrm{Mg}, \mathrm{Na}, \mathrm{Zn}, \mathrm{Fe}$ and $\mathrm{Mn}$. Leaf nutrient levels determination included $\mathrm{N}, \mathrm{P}, \mathrm{K}, \mathrm{Ca}, \mathrm{Mg}, \mathrm{Zn}, \mathrm{Fe}$ and $\mathrm{Mn}$. Yield per tree was recorded for one season and quality parameters were measured for fruits sampled from representative trees. Results indicated that, soil texture of the orchards ranged between clay and clay loam. Although the orchards were receiving the same cultural practices, yet, they were significantly different in some soil and plant constituents. All orchards were non-saline and non-sodic of slightly to moderately alkaline reaction. Soil and plant were deficient in nitrogen and zinc with high leaf phosphorus, magnesium, iron and deficit calcium levels. Mean fruit yield ranged between 0.93 and 7.76 tons ha ${ }^{-1}$ with an average of 4.35 tons ha $^{-1}$, which is considered lower than world average. A low \% TSS (total soluble solids), low ascorbic acid content and high \% green color on fruit surface are evidents of low quality fruits. The low yield and poor quality fruits in this area were attributed to unsuitable soil conditions and the poor management practices, including lack of fertilizer application.
\end{abstract}

\section{INTRODUCTION}

Most of the highly fertile lands along the Nile and its tributaries in Sudan have been brought under cultivation. Therefore, the Central Clay Plain, which includes the large national schemes such as Gezira, Suki, Rahad, Blue Nile and New Halfa schemes, is the area where the national strategy of citrus expansion is thought of (Sidahmed and Geneif, 1984). Citrus trees grown in this area look stunted with low yield and poor quality fruits (Hamid, 1995; Wardowiski, 1990). Many factors can be suspected in this situation. These factors may include the use of local low yielding yield and poor quality fruits (Hamid, 1995; Wardowiski, 1990). Many factors can be suspected in this situation. These factors may include the use of local low yielding cultivars, poor cultural practices and soil fertility factors. No studies were conducted to investigate the fertility status of citrus trees grown in this area.

Therefore, the main objective of the present survey is to study the fertility status of citrus orchards of Sudan Central Clay Plain in view of correlating soil conditions and plant nutrient status with fruit yield and quality.

\section{MATERIALS AND METHODS}

Eight orchards of 18 years old Foster grapefruit (Citrus paradisi Macf.) trees budded on Sour orange (Citrus aurantium L.) were surveyed in Sennar area $\left(13^{\circ} 37^{\prime} \mathrm{N}, 33^{\circ} 37^{\prime} \mathrm{E}\right)$ representing Sudan Central Clay Plain. The cultural practices carried out in these orchards were similar as far as weed 
control, pruning and fertilizer application are concerned. Selected trees, free of the major pests and diseases such as scale insets, leaf minor, lemon butter fly and gummosis, were used in this study.

A randomized complete block design was adopted. Each orchard was divided into three blocks according to their vicinity to the Blue Nile bank. Block 1 was towards the Nile ( $1^{\text {st }}$ terrace), block 11 in the middle ( $2^{\text {nd }}$ terrace) and block 111 was away from the Blue Nile bank ( $3^{\text {rd }}$ terrace). Total number of experimental units was 24 .

\section{Sampling and analysis}

In each orchard, soil samples were obtained from three sites representing the three blocks. The sites were 40-50 meters apart in the west-east direction. Soil samples were collected from the feeder root zone at two depths (0-30 and 30-60 $\mathrm{cm}$ ) in each site according to Noling (2003). Soil samples were air dried ground and sieved to pass a $2 \mathrm{~mm}$ sieve.

Standard analytical procedures were used for soil physical and chemical properties (Grigal, 1974; Gough et al 1980 and Tandon, 1993). Soil samples were analyzed for particle size distribution, $\mathrm{pH}$ (paste), ECe, SAR, N, P, K, Ca, Mg, Na, $\mathrm{Zn}, \mathrm{Fe}$ and Mn contents.

Three healthy trees around each sampling site were selected. Leaf samples were collected from non-fruiting terminals in August which appeared to be the most stable sampling period to be related to yield and quality. Hundred leaves per tree were collected and analyzed according to standard methods (Obreza et al 1999).

Leaf analysis included N, P, K, Ca, Mg, Zn, Fe and $\mathrm{Mn}$ contents.

Crop yield consisted of all picks (April to August). Yield was recorded in tons $\mathrm{ha}^{-1}$.

Four fruits of mean size per tree were randomly sampled in August for quality studies. The following parameters which were determined for fruit samples according to standard procedure (Wardowiski, 1990) were used for quality evaluation. These parameters included fruit diameter, peel thickness, fruit juice, total soluble solids (\% TSS), titrable acidity (\% TA) and ascorbic acid (vit$\operatorname{amin} \mathrm{C})$.

Statistical analysis was carried out using Gomez and Gomez (1984) and Irristat (2005) computer software method.

\section{RESULTS AND DISCUSSION}

Data obtained from the survey revealed that, soil texture of the orchards ranged between clay and clay loam (Table 1). Although the orchards were significantly different at $(P \geq 0.05)$ and $(P \geq$ 0.01 ) in sodium adsorption ratio in surface and sub surface samples, respectively, yet, all orchards were non-saline, non-sodic of slightly to moderately alkaline reaction $(\mathrm{pH}=7.3-7.8)$ according to Ghassemi et al (1995) (Table 2). Table (3a) showed soil total $\mathrm{N}$, available $\mathrm{P}$ and soluble $\mathrm{K}$. The orchards were significantly different at $(P \geq 0.01)$ and $(P \geq 0.05)$ in $N$ of surface and subsurface, respectively and significantly different $(P \geq 0.01)$ in soil $\mathrm{K}$ in the two depths. Table ( $\mathbf{a} \mathbf{a} \mathbf{b})$ on the other hand, indicated that the orchards were significantly different at $(P \geq 0.05)$ in $\mathrm{Mg}$ of surface and $\mathrm{Na}$ in the two depths. Table (4) depicted significant difference $(P \geq 0.05)$ in soil $M n$ of the surface. However, the orchards were of deficit soil nitrogen and zinc levels. Tables (5 \& 6) illustrated plant nutrient levels. The orchards were significantly different at $(P \geq 0.01)$ in leaf $N$ and $(P \geq 0.05)$ in leaf $\mathrm{K}, \mathrm{Mg}$ and $\mathrm{Mn}$. According to He et al (2003) and Obreza et al (1992), the orchards were deficient in leaf nitrogen, calcium and zinc with high levels of phosphorus, magnesium and iron. Tables (7a \&7b) showed mean fruit yield and quality parameters of the surveyed orchards. The orchards were significantly different at $(P \geq 0.01)$ in yield, peel thickness and \% green color on fruit surface and significantly different $(P \geq 0.05)$ in juice \% by weight and mean fruit weight. Mean yield ranged between 0.93 and 7.76 tons ha $^{-1}$ with an average of 4.35 tons ha $^{-1}$ which is considered lower than average of worlds similar areas (10.7 tons ha ${ }^{-1}$ ) (FASS, 2000). Surveyed orchards were of low $\%$ TSS contents, low ascorbic acid and of high \% green color on fruit surface according to standard levels (Wardowiski, 1990). The low yield and poor quality fruits may be due to the increased clay contents in the root zone which may have contributed to very high water contents, hence, poor aeration that might have resulted in decreased or low root density and unfavorable growth conditions. Poor aeration conditions may also lead to upset of nutrients absorption and formation of toxic compounds resulting from low decomposition of organic matter such as lactic, butyric and citric acids as reported by Brady and Weil (2000).In addition, the high clay contents may increase $\mathrm{Zn}$ and other micronutrients fixation. Under such conditions, the capacity of the feeder 
Table 1. Surface and subsurface soil particle size distribution and textural class of the surveyed 8 Foster grapefruit orchards in Sennar area

\begin{tabular}{|c|c|c|c|c|c|c|c|c|}
\hline \multirow[b]{2}{*}{ Orchard } & \multicolumn{4}{|c|}{$0-30 \mathrm{~cm}$} & \multicolumn{4}{|c|}{$30-60 \mathrm{~cm}$} \\
\hline & Sand \% & Silt \% & Clay \% & $\begin{array}{c}\text { Texture } \\
\text { Class }\end{array}$ & Sand \% & Silt \% & Clay\% & $\begin{array}{c}\text { Texture } \\
\text { Class }\end{array}$ \\
\hline 1 & 37 & 24 & 39 & $\begin{array}{l}\text { Clay } \\
\text { loam }\end{array}$ & 38 & 22 & 40 & $\begin{array}{l}\text { clay } \\
\text { loam }\end{array}$ \\
\hline 2 & 37 & 14 & 49 & Clay & 38 & 14 & 48 & Clay \\
\hline 3 & 25 & 28 & 47 & $\begin{array}{l}\text { Clay } \\
\text { loam }\end{array}$ & 34 & 25 & 41 & $\begin{array}{l}\text { Clay } \\
\text { loam }\end{array}$ \\
\hline 4 & 32 & 25 & 43 & $\begin{array}{l}\text { Clay } \\
\text { loam }\end{array}$ & 34 & 23 & 43 & $\begin{array}{l}\text { Clay } \\
\text { loam }\end{array}$ \\
\hline 5 & 36 & 25 & 39 & $\begin{array}{l}\text { Clay } \\
\text { loam }\end{array}$ & 27 & 24 & 49 & $\begin{array}{l}\text { Clay } \\
\text { loam }\end{array}$ \\
\hline 6 & 26 & 28 & 46 & Clay & 25 & 25 & 50 & Clay \\
\hline 7 & 30 & 24 & 46 & Clay & 27 & 19 & 54 & Clay \\
\hline 8 & 37 & 17 & 46 & Clay & 39 & 12 & 49 & Clay \\
\hline
\end{tabular}

Table 2. Some soil chemical properties of the surveyed 8 Foster grapefruit orchards in Sennar area

\begin{tabular}{|l|c|c|c|c|c|c|}
\hline \multirow{2}{*}{ Orchard } & \multicolumn{2}{|c|}{$\mathrm{pH}$} & \multicolumn{2}{c|}{ ECe $\left(\mathrm{dSm}^{-1}\right)$} & \multicolumn{2}{c|}{ SAR } \\
\cline { 2 - 7 } & $0-30 \mathrm{~cm}$ & $30-60 \mathrm{~cm}$ & $0-30 \mathrm{~cm}$ & $30-60 \mathrm{~cm}$ & $0-30 \mathrm{~cm}$ & $30-60 \mathrm{~cm}$ \\
\hline 1 & 7.3 & 7.3 & $0.49 \mathrm{a}$ & $0.72 \mathrm{a}$ & $0.23 \mathrm{ab}$ & $0.20 \mathrm{ab}$ \\
2 & 7.5 & 7.6 & $0.48 \mathrm{a}$ & $0.70 \mathrm{a}$ & $0.41 \mathrm{a}$ & $0.42 \mathrm{a}$ \\
3 & 7.8 & 7.6 & $0.42 \mathrm{a}$ & $0.81 \mathrm{a}$ & $0.12 \mathrm{~b}$ & $0.14 \mathrm{~b}$ \\
4 & 7.7 & 7.7 & $0.47 \mathrm{a}$ & $0.46 \mathrm{a}$ & $0.13 \mathrm{~b}$ & $0.15 \mathrm{~b}$ \\
5 & 7.7 & 7.7 & $0.66 \mathrm{a}$ & $0.88 \mathrm{a}$ & $0.14 \mathrm{~b}$ & $0.12 \mathrm{~b}$ \\
6 & 7.4 & 7.5 & $0.50 \mathrm{a}$ & $0.60 \mathrm{a}$ & $0.13 \mathrm{~b}$ & $0.12 \mathrm{~b}$ \\
7 & 7.5 & 7.8 & $0.69 \mathrm{a}$ & $0.64 \mathrm{a}$ & $0.23 \mathrm{ab}$ & $0.41 \mathrm{a}$ \\
8 & 7.4 & 7.5 & $0.45 \mathrm{a}$ & $0.38 \mathrm{a}$ & $0.18 \mathrm{~b}$ & $0.20 \mathrm{ab}$ \\
\hline F-value & & & $\mathrm{NS}$ & $\mathrm{NS}$ & $*$ & $* *$ \\
\hline SE & & & 0.29 & 0.19 & 0.76 & 0.07 \\
\hline LSD & & & 0.62 & 0.41 & 0.16 & 0.15 \\
\hline
\end{tabular}

-Each value is a mean of 3 replicates

-NS = Not significant

-Means within a column followed by same letters are significantly different 
Table 3a. Soil total nitrogen, available phosphorus and soluble potassium contents of the surveyed 8 Foster grapefruit orchards in Sennar area

\begin{tabular}{|l|l|l|l|l|l|c|}
\hline \multirow{3}{*}{ Orchard } & \multicolumn{2}{|c|}{$\begin{array}{c}\text { Total } \mathrm{N} \\
(\%)\end{array}$} & \multicolumn{2}{c|}{$\begin{array}{c}\text { Available } \mathrm{P} \\
(\mathrm{ppm})\end{array}$} & \multicolumn{2}{c|}{$\begin{array}{c}\text { Soluble K } \\
(\mathrm{meq} / \mathrm{L})\end{array}$} \\
\cline { 2 - 7 } & $0-30 \mathrm{~cm}$ & $30-60 \mathrm{~cm}$ & $0-30 \mathrm{~cm}$ & $30-60 \mathrm{~cm}$ & $0-30 \mathrm{~cm}$ & $30-60 \mathrm{~cm}$ \\
\hline 1 & $0.016 \mathrm{~b}$ & $0.062 \mathrm{~b}$ & $1.9 \mathrm{a}$ & $1.23 \mathrm{a}$ & $0.13 \mathrm{bc}$ & $0.02 \mathrm{a}$ \\
2 & $0.026 \mathrm{~d}$ & $0.036 \mathrm{~b}$ & $0.41 \mathrm{a}$ & $2.4 \mathrm{a}$ & $0.01 \mathrm{~d}$ & $0.01 \mathrm{~b}$ \\
3 & $0.070 \mathrm{ab}$ & $0.045 \mathrm{ab}$ & $4.79 \mathrm{a}$ & $3.92 \mathrm{a}$ & $0.16 \mathrm{ab}$ & $0.19 \mathrm{a}$ \\
4 & $0.090 \mathrm{a}$ & $0.074 \mathrm{a}$ & $5.05 \mathrm{a}$ & $4.0 \mathrm{a}$ & $0.26 \mathrm{a}$ & $0.23 \mathrm{a}$ \\
5 & $0.070 \mathrm{ab}$ & $0.074 \mathrm{a}$ & $4.13 \mathrm{a}$ & $4.7 \mathrm{a}$ & $0.23 \mathrm{c}$ & $0.04 \mathrm{~b}$ \\
6 & $0.093 \mathrm{a}$ & $0.049 \mathrm{~b}$ & $4.88 \mathrm{a}$ & $2.4 \mathrm{a}$ & $0.14 \mathrm{~b}$ & $0.01 \mathrm{~b}$ \\
7 & $0.057 \mathrm{bc}$ & $0.035 \mathrm{~b}$ & $1.5 \mathrm{a}$ & $0.92 \mathrm{a}$ & $0.02 \mathrm{c}$ & $0.01 \mathrm{~b}$ \\
8 & $0.032 \mathrm{c}$ & $0.024 \mathrm{~b}$ & $0.63 \mathrm{a}$ & $1.28 \mathrm{a}$ & $0.01 \mathrm{~d}$ & $0.01 \mathrm{~b}$ \\
\hline Sig.level & $\star *$ & $*$ & $\mathrm{NS}$ & $\mathrm{NS}$ & $* *$ & $* *$ \\
\hline SE & 0.01 & 0.014 & 1.11 & 1.92 & 0.05 & 0.03 \\
\hline LSD & 0.021 & 0.03 & 2.38 & 4.11 & 0.1 & 0.06 \\
\hline
\end{tabular}

-Each value is a mean of 3 replicates

-NS=Not significantly different

- Means followed by same letters are not significantly different.

Table $3 b$. Soil soluble calcium, magnesium and sodium levels (meq/L) of the surveyed 8 Foster grapefruit orchards in Sennar area

\begin{tabular}{|l|c|c|c|c|c|c|}
\hline \multirow{2}{*}{ Orchard } & \multicolumn{2}{|c|}{ Ca } & \multicolumn{2}{c|}{$\mathrm{Mg}$} & \multicolumn{2}{c|}{$\mathrm{Na}$} \\
\cline { 2 - 7 } & $0-30 \mathrm{~cm}$ & $30-60 \mathrm{~m}$ & $0-30 \mathrm{~cm}$ & $30-60 \mathrm{~cm}$ & $0-30 \mathrm{~cm}$ & $30-60 \mathrm{~cm}$ \\
\hline 1 & $3.33 \mathrm{a}$ & $6.5 \mathrm{a}$ & $3.33 \mathrm{a}$ & $3.67 \mathrm{a}$ & $0.42 \mathrm{ab}$ & $0.44 \mathrm{ab}$ \\
2 & $3.0 \mathrm{a}$ & $2.5 \mathrm{a}$ & $3.0 \mathrm{a}$ & $2.67 \mathrm{a}$ & $0.73 \mathrm{a}$ & $0.69 \mathrm{a}$ \\
3 & $4.0 \mathrm{a}$ & $4.5 \mathrm{a}$ & $2.33 \mathrm{ab}$ & $4.67 \mathrm{a}$ & $0.22 \mathrm{~b}$ & $0.29 \mathrm{~b}$ \\
4 & $4.83 \mathrm{a}$ & $3.5 \mathrm{a}$ & $1.17 \mathrm{~b}$ & $1.70 \mathrm{a}$ & $0.22 \mathrm{~b}$ & $0.22 \mathrm{~b}$ \\
5 & $5.17 \mathrm{a}$ & $4.83 \mathrm{a}$ & $1.5 \mathrm{~b}$ & $2.33 \mathrm{a}$ & $0.25 \mathrm{~b}$ & $0.22 \mathrm{~b}$ \\
6 & $3.0 \mathrm{a}$ & $4.17 \mathrm{a}$ & $3.0 \mathrm{a}$ & $2.33 \mathrm{a}$ & $0.22 \mathrm{~b}$ & $0.22 \mathrm{~b}$ \\
7 & $4.17 \mathrm{a}$ & $2.17 \mathrm{a}$ & $1.5 \mathrm{~b}$ & $0.83 \mathrm{a}$ & $0.37 \mathrm{~b}$ & $050 \mathrm{a}$ \\
8 & $2.5 \mathrm{a}$ & $3.0 \mathrm{a}$ & $3.5 \mathrm{a}$ & $5.0 \mathrm{a}$ & $0.31 \mathrm{~b}$ & $0.34 \mathrm{~b}$ \\
\hline Sig.level & $\mathrm{NS}$ & $\mathrm{NS}$ & $*$ & $\mathrm{NS}$ & $*$ & $*$ \\
\hline SE & 1.32 & 1.7 & 0.65 & 1.39 & 0.14 & 0.12 \\
\hline LSD & 2.82 & 3.65 & 1.4 & 2.98 & 0.3 & 0.26 \\
\hline
\end{tabular}

-Each value is a mean of 3 replicates

-NS=Not significantly different

- Means followed by same letters are not significantly different 
Table 4. Soil EDTA extractable zinc, iron and manganese levels (ppm) of the surveyed 8 Foster grapefruit orchards in Sennar area

\begin{tabular}{|l|c|c|c|c|c|c|}
\hline \multirow{2}{*}{ Orchard } & \multicolumn{2}{|c|}{ Zn } & \multicolumn{2}{c|}{ Fe } & \multicolumn{2}{c|}{ Mn } \\
\cline { 2 - 7 } & $0-30 \mathrm{~cm}$ & $30-60 \mathrm{~cm}$ & $0-30 \mathrm{~cm}$ & $30-60 \mathrm{~cm}$ & $0-30 \mathrm{~cm}$ & $30-60 \mathrm{~cm}$ \\
\hline 1 & $1.61 \mathrm{a}$ & $1.32 \mathrm{a}$ & $14.6 \mathrm{a}$ & $21.5 \mathrm{a}$ & $6.53 \mathrm{a}$ & $6.54 \mathrm{bc}$ \\
2 & $2.01 \mathrm{a}$ & $1.67 \mathrm{a}$ & $13.0 \mathrm{a}$ & $14.9 \mathrm{a}$ & $5.07 \mathrm{a}$ & $6.58 \mathrm{~b}$ \\
3 & $0.84 \mathrm{a}$ & $0.99 \mathrm{a}$ & $10.7 \mathrm{a}$ & $12.3 \mathrm{a}$ & $6.76 \mathrm{a}$ & $6.92 \mathrm{ab}$ \\
4 & $2.67 \mathrm{a}$ & $2.60 \mathrm{a}$ & $18.6 \mathrm{a}$ & $32.1 \mathrm{a}$ & $7.36 \mathrm{a}$ & $7.40 \mathrm{a}$ \\
5 & $2.53 \mathrm{a}$ & $1.75 \mathrm{a}$ & $14.6 \mathrm{a}$ & $11.6 \mathrm{a}$ & $6.81 \mathrm{a}$ & $6.73 \mathrm{~b}$ \\
6 & $1.55 \mathrm{a}$ & $1.34 \mathrm{a}$ & $17.7 \mathrm{a}$ & $20.6 \mathrm{a}$ & $6.84 \mathrm{a}$ & $6.93 \mathrm{a}$ \\
7 & $2.10 \mathrm{a}$ & $1.00 \mathrm{a}$ & $15.8 \mathrm{a}$ & $16.8 \mathrm{a}$ & $6.72 \mathrm{a}$ & $6.54 \mathrm{bc}$ \\
8 & $1.45 \mathrm{a}$ & $1.33 \mathrm{a}$ & $25.1 \mathrm{a}$ & $17.7 \mathrm{a}$ & $6.77 \mathrm{a}$ & $6.28 \mathrm{c}$ \\
\hline F.value & $\mathrm{NS}$ & $\mathrm{NS}$ & $\mathrm{NS}$ & $\mathrm{NS}$ & $\mathrm{NS}$ & $*$ \\
\hline SE \pm & 0.94 & 0.37 & 3.72 & 3.6 & 0.73 & 0.23 \\
\hline LSD & 2.01 & 1.33 & 8.1 & 7.72 & 1.57 & 0.49 \\
\hline
\end{tabular}

-Each value is a mean of 3replicates

-NS= Not significant

-Means followed by same letters are not significantly different.

Table 5. Leaf macronutrient levels (\%) of the surveyed 8 Foster grapefruit orchards in Sennar area

\begin{tabular}{|l|c|c|c|c|c|}
\hline \multicolumn{1}{|c|}{ Orchard } & $\mathrm{N}$ & $\mathrm{P}$ & $\mathrm{K}$ & $\mathrm{Ca}$ & $\mathrm{Mg}$ \\
\hline 1 & $1.93 \mathrm{a}$ & $0.60 \mathrm{a}$ & $0.36 \mathrm{~b}$ & $2.7 \mathrm{a}$ & $1.02 \mathrm{a}$ \\
2 & $1.83 \mathrm{~b}$ & $0.62 \mathrm{a}$ & $0.44 \mathrm{~b}$ & $3.2 \mathrm{a}$ & $1.00 \mathrm{a}$ \\
3 & $1.91 \mathrm{a}$ & $0.67 \mathrm{a}$ & $0.50 \mathrm{a}$ & $2.9 \mathrm{a}$ & $1.01 \mathrm{a}$ \\
4 & $1.80 \mathrm{~b}$ & $0.63 \mathrm{a}$ & $0.60 \mathrm{a}$ & $2.9 \mathrm{a}$ & $0.51 \mathrm{~b}$ \\
5 & $1.75 \mathrm{~b}$ & $0.62 \mathrm{a}$ & $0.54 \mathrm{a}$ & $2.7 \mathrm{a}$ & $0.56 \mathrm{~b}$ \\
6 & $1.57 \mathrm{~b}$ & $0.59 \mathrm{a}$ & $0.52 \mathrm{a}$ & $2.7 \mathrm{a}$ & $0.69 \mathrm{~b}$ \\
7 & $1.57 \mathrm{~b}$ & $0.51 \mathrm{a}$ & $0.35 \mathrm{~b}$ & $2.5 \mathrm{a}$ & $0.82 \mathrm{ab}$ \\
8 & $1.29 \mathrm{~b}$ & $0.56 \mathrm{a}$ & $0.43 \mathrm{~b}$ & $3.2 \mathrm{a}$ & $0.63 \mathrm{~b}$ \\
\hline F.value & $* *$ & $\mathrm{NS}$ & $*$ & $\mathrm{NS}$ & $*$ \\
\hline SE \pm & 0.03 & 0.06 & 0.07 & 0.03 & 0.27 \\
\hline LSD & 0.06 & 0.14 & 0.15 & 0.64 & 0.58 \\
\hline CV\% & 1.74 & 10.5 & 10.2 & 10.5 & 13.8 \\
\hline
\end{tabular}

-Each value is a mean of 3 replicate -NS= Not significant

-Means followed by same letters are not significantly different 
Table 6. Leaf micronutrient levels (ppm) of the surveyed 8 Foster grapefruit orchards in Sennar area

\begin{tabular}{|l|c|c|c|}
\hline \multicolumn{1}{|c|}{ Orchard } & Zn & Fe & Mn \\
\hline 1 & $16.2 \mathrm{a}$ & $233.4 \mathrm{a}$ & $23.0 \mathrm{~b}$ \\
2 & $19.4 \mathrm{a}$ & $255.6 \mathrm{a}$ & $24.5 \mathrm{~b}$ \\
3 & $13.8 \mathrm{a}$ & $220.4 \mathrm{a}$ & $20.9 \mathrm{~b}$ \\
4 & $16.1 \mathrm{a}$ & $144.9 \mathrm{a}$ & $19.6 \mathrm{~b}$ \\
5 & $12.2 \mathrm{a}$ & $169.9 \mathrm{a}$ & $19.1 \mathrm{~b}$ \\
6 & $15.5 \mathrm{a}$ & $190.8 \mathrm{a}$ & $22.2 \mathrm{~b}$ \\
7 & $15.5 \mathrm{a}$ & $139.9 \mathrm{a}$ & $16.6 \mathrm{~b}$ \\
8 & $13.1 \mathrm{a}$ & $165.7 \mathrm{a}$ & $36.1 \mathrm{a}$ \\
\hline F.value & $\mathrm{NS}$ & $\mathrm{NS}$ & $*$ \\
\hline SE \pm & 4.04 & 45.9 & 4.15 \\
\hline LSD & 8.67 & 98.5 & 8.9 \\
\hline CV\% & 26.5 & 24.2 & 18.3 \\
\hline
\end{tabular}

-Each value is a mean of 3 replicate -NS= Not significant

-Means followed by same letters are not significantly different

Table 7a. Mean fruit yield and quality parameters of the surveyed 8 Foster grapefruit orchards in Sennar area

\begin{tabular}{|l|c|c|c|c|c|c|}
\hline Orchard & $\begin{array}{c}\text { Yield } \\
(\text { tonha-1 }\end{array}$ & $\begin{array}{c}\text { Fruit } \\
\text { diameter } \\
(\mathrm{cm})\end{array}$ & $\begin{array}{c}\text { Fruit } \\
\text { length/width }\end{array}$ & $\begin{array}{c}\text { Peel } \\
\text { thickness } \\
(\mathrm{mm})\end{array}$ & $\begin{array}{c}\text { \% green } \\
\text { color on fruit } \\
\text { surface }\end{array}$ & $\begin{array}{c}\text { Juice \% } \\
\text { by } \\
\text { weight }\end{array}$ \\
\hline 1 & $7.76 \mathrm{a}$ & $7.9 \mathrm{a}$ & $0.92 \mathrm{a}$ & $7.9 \mathrm{c}$ & $35.7 \mathrm{~d}$ & $26.1 \mathrm{~b}$ \\
3 & $0.93 \mathrm{~d}$ & $6.7 \mathrm{a}$ & $1.01 \mathrm{a}$ & $12.9 \mathrm{ab}$ & $75.0 \mathrm{ab}$ & $16.5 \mathrm{~b}$ \\
4 & $3.8 \mathrm{~b}$ & $6.8 \mathrm{a}$ & $0.97 \mathrm{a}$ & $7.54 \mathrm{c}$ & $58.3 \mathrm{bc}$ & $26.3 \mathrm{a}$ \\
5 & $4.26 \mathrm{~b}$ & $6.5 \mathrm{a}$ & $0.94 \mathrm{a}$ & $7.1 \mathrm{~cd}$ & $40.1 \mathrm{~d}$ & $31.1 \mathrm{a}$ \\
6 & $4.45 \mathrm{~b}$ & $7.2 \mathrm{a}$ & $0.92 \mathrm{a}$ & $8.3 \mathrm{c}$ & $6.0 \mathrm{~b}$ & $25.9 \mathrm{a}$ \\
7 & $3.73 \mathrm{~b}$ & $7.5 \mathrm{a}$ & $0.94 \mathrm{a}$ & $9.7 \mathrm{bc}$ & $48.7 \mathrm{~cd}$ & $32.0 \mathrm{a}$ \\
8 & $3.18 \mathrm{bc}$ & $6.2 \mathrm{a}$ & $0.93 \mathrm{a}$ & $6.0 \mathrm{~d}$ & $35.0 \mathrm{~d}$ & $25.7 \mathrm{ab}$ \\
\hline F.value & $1.65 \mathrm{c}$ & $7.2 \mathrm{a}$ & $1.00 \mathrm{a}$ & $13.3 \mathrm{a}$ & $81.0 \mathrm{a}$ & $20.5 \mathrm{~b}$ \\
\hline SE \pm & $* *$ & $\mathrm{NS}$ & $\mathrm{NS}$ & $* *$ & $* *$ & $*$ \\
\hline LSD & 0.8 & 0.64 & 0.03 & 1.46 & 7.44 & 4.15 \\
\hline CV\% & 21.5 & 9.1 & 3.4 & 16.1 & 13.7 & 16.3 \\
\hline
\end{tabular}

1 hectare $=156$ trees

-Each value is a mean of 9 replicates

- Mean values with different subscript letters are significantly different. 
Table 7b. Quality parameters of the surveyed 8 Foster grapefruit orchards in Sennar area

\begin{tabular}{|c|c|c|c|c|c|}
\hline Orchard & TSS (\%) & $(\% \mathrm{TA})$ & $\begin{array}{c}\text { Maturity ratio } \\
\text { (TSS/TA) }\end{array}$ & $\begin{array}{c}\text { Ascorbic acid } \\
\text { (vit C) } \\
\text { (mg/100cc) }\end{array}$ & $\begin{array}{l}\text { Mean fruit } \\
\text { weight } \\
\text { (g) }\end{array}$ \\
\hline 1 & $9.3 a$ & $0.98 a$ & $10.1 \mathrm{a}$ & $32.9 a$ & $281.7 a$ \\
\hline 2 & $8.8 a$ & $1.00 \mathrm{a}$ & $8.8 a$ & $41.4 a$ & $151.3 b$ \\
\hline 3 & $10.4 a$ & $1.16 a$ & $9.0 \mathrm{a}$ & $39.6 a$ & $170.7 b$ \\
\hline 4 & $10.8 \mathrm{a}$ & $0.96 a$ & $12.1 \mathrm{a}$ & $35.5 a$ & $277.7 a$ \\
\hline 5 & $11.0 \mathrm{a}$ & $1.12 \mathrm{a}$ & $9.8 a$ & $45.3 a$ & $268.7 a$ \\
\hline 6 & $9.1 a$ & $1.21 \mathrm{a}$ & $7.6 a$ & $36.0 \mathrm{a}$ & $292.7 a$ \\
\hline 7 & $11.7 a$ & $1.10 a$ & $12.5 a$ & $45.1 \mathrm{a}$ & $216.7 a$ \\
\hline 8 & $8.5 a$ & $0.97 a$ & $8.9 a$ & $39.0 a$ & 186.3ab \\
\hline F.value & NS & NS & NS & NS & * \\
\hline $\mathrm{SE} \pm$ & 1.05 & 0.18 & 2.26 & 6.74 & 47.5 \\
\hline LSD & 2.25 & 0.39 & 4.85 & 14.5 & 101.9 \\
\hline CV\% & 10.6 & 17 & 22.9 & 17.1 & 20.7 \\
\hline
\end{tabular}

-Each value is a mean of 9 replicates

- Mean values with different subscript letters are significantly different.

TSS $=$ Total soluble solids (mainly sugars)

$\% \mathrm{TA}=$ Percent titrable acidity

roots to take up nutrients can be reduced. Similar results were reported by Wiedendfeld et al (1982). Besides, the trees are subject to short supply of the most necessary nutrients. This may be related to the fact that, most of these soils are inherently deficient in nitrogen (Blockhuis, 1993) and of high $\mathrm{pH}$ values which adversely affect the availability of micronutrients in particular (Sims, 1986). These results were supported by the findings of Hamid (1995). He related the low yield and poor quality of fruit crops in this area to micronutrients deficiency and that was reflected by tree response to micronutrients application. In addition, Ali (1998) suggested that this low yield is due to absence of proper fertilization programs, removal of leaf litters from surface and use of herbicides or hand hoeing in Central Sudan depriving the soil from any organic residues which may aggravate the situation of plant nutrition. However, the conditions prevailing in these orchards are conducive to imbalanced nutritional status within the plant tissue indicated by low leaf nitrogen, calcium, zinc and high phosphorus, magnesium and iron levels. Consequently, tree growth, yield and quality were adversely affected. It is well known that nitrogen and zinc are essential to plant growth from early stages to har- vest by affecting development of root system, leaves, flowers and fruits (Zekri and Obreza, 2008). The low quality may also be related to calcium deficiency levels in the plant tissue. It is known that fruits and vegetables are affected in quality by conditions of localized calcium deficiencies. Shortage of calcium during fruit development was found to cause fruit disorders because calcium reserves are not mobile (Zekri and Obreza, 2009).

It can be concluded that, the high clay contents and the relatively high soil $\mathrm{pH}$ values in some orchards may be the cause of poor root density and low permeability to water and air, which, in turn adversely affect availability and uptake of plant nutrients. Besides, lack of proper management practices including irrigation, pruning and fertilization could be one of the major factors of the stunted growth, low yield and poor quality of citrus grown in Sudan Central Clay Plain. Therefore, the present study suggested proper management practices to improve soil physical and chemical conditions for better growth, such as keeping and incorporation of leaf litters in soil and application of organic and inorganic fertilizers frequently. 


\section{REFERENCES}

Ali, N.A. (1998). Organic Fertilization in Sudan. pp. 125-132. $1^{\text {st }}$ Collogium, Land and Water Research Center, Agricultural Research Corporation (ARC), Wad Medani, Sudan.

Blockhuis, W.A. (1993). Vertisols in the Central Clay Plain of the Sudan. Doctoral Thesis. Wageningen, Nothernland.

Brady, N.C. and R.R. Weil (2000). Elements of the Nature and Properties of Soils. 12 ${ }^{\text {th }}$ Edition. Prentice-Hall, London, UK.

Florida Agricultural Statistics Service (FASS). (2000). Citrus 1999-2000 Summary- Production, price, and value production by counties and per tree. FASS Historic Reports. September, 2000, Orlando, FL.,USA.

Ghassemi, F.; A.J. Jakeman and H.A. Nix (1995). Salinization of Land and Water Resources: Human causes, extent, management and case studies. Centre for Resource and Environmental Studies, Canberra, ACT, Australia.

Gomez, K.A. and A.A. Gomez (1984). Statistical Procedures for Agricultural Research. $2^{\text {nd }}$ Ed. John Wiley and Sons, Inc., New York.

Gough, L.P.; J.M. McNeal and R.C. Severson (1980). Predicting native plant copper, iron, manganese and zinc levels using DTPA and EDTA soil extractants, Northern Great Plains. Soil Sci Soc. of Am. J. 44(5): 1030-1036.

Grigal, D.F. (1974). Notes on the hydrometer method of particle size analysis. Minnesota for Res. Notes. 245., USA.

Hamid, G.A. (1995). Nutritional Status of Fruit Crops in Central States of the Sudan and its improvement with special reference to micronutrients. Proc $2^{\text {nd }}$ Sudanese-Egyptian Workshop on Micronutrients and Plant Nutrition, pp. 171 - 181. Wad Medani-Sudan. Eds. M.M. El-Fouly and A.I. Rezk.

He, Z.L.; D.V. Calvert; A.K. Alva; D.J. Banks and Y.C. Li (2003). Thresholds of Leaf Nitrogen for
Optimum Fruit Production and Quality in Grapefruit. Soil Sci. Soc. of Amer. J. 67: 583-588.

Irristat (2005). Irristat for Windows. Version 5. Tutorial Manual Biometrics and Bioinformatics Unit. International Rice Research Institute. Manilla, Philippines.

Noling, J.W. (2003). Citrus Root Growth and Soil Management. Citrus Research and Education Center (CREC), Insitute of Food and Agricultural Sciences, University of Florida, Gainsville FL 32611(pub.)

Obreza, T.A.; A.K. Alva; E.A. Hanlon and R.E. Rouse (1999). Citrus grove leaf- tissue and soil testing: sampling, analysis and interpretation. Univ. of Florida. Coop. Ext. Ser. Bull. SL115: 1-4.

Sidahmed, O.A. and A.A. Geneif (1984). Performance of citrus in the irrigated heavy clay soils of Central Sudan. I. Lemon. Acta. Horticulture (143): 247-255.

Sims, J.T. (1986). Soil pH effects on the distribution and plant availability of manganese, copper and zinc. Soil Sci. Soc. Amer. J. 50(2): 367-373.

Tandon, H.L.S. (1993).Methods of Analysis of Soils, Plants, Waters and Fertilizers. Fertilizers Development and Consultation Organization. New Dehi (India).

Wardowiski, W.F. (1990). Citrus Quality Control Assessment Methodology. Citrus Research and Education Center, University of Florida, Lake Alfred, FL.

Wiedenfeld, R.P.; C.G. Lyons and R.E. Rose (1982). Effect of soil texture on citrus root development and tree vigor. Journal of the Rio Grande Valley, Horticultural Society 35: 65-78.

Zekri, M. and T.A. Obreza (2008). Plant Nutrients for Citrus Trees. Institute of Food and Agricultural Sciences, University of Florida, Gainesville 32611-0290. (Pub.)

Zekri, M. and T.A. Obreza (2009). Macronutrients Deficiencies in Citrus: Calcium, Magnesium and Sulfur. Institute of Food and Agricultural Sciences, University of Florida, Gainesville, 326110290. (Pub.). 